\title{
Ticagrelor and preconditioning in patients with stable coronary artery disease (TAPER- S): a randomized pilot clinical trial
}

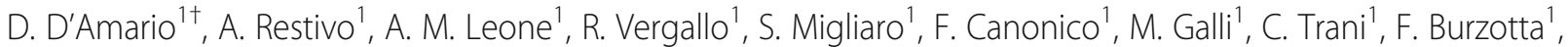 \\ C. Aurigemma ', G. Niccoli', A. Buffon ${ }^{1}$, R. A. Montone' ${ }^{1}$, A. Flex ${ }^{1}$, F. Franceschi ${ }^{1}$, G. Tinelli', U. Limbruno ${ }^{2}$, \\ F. Francese' ${ }^{1}$, I. Ceccarelli ${ }^{1}$, J. A. Borovac ${ }^{3}$, I. Porto ${ }^{4 * \dagger}$ and F. Crea ${ }^{1 *}$
}

\begin{abstract}
Background: Ticagrelor is a reversibly binding, direct-acting, oral, $\mathrm{P}_{2} \mathrm{Y}_{12}$ antagonist used for the prevention of atherothrombotic events in patients with coronary artery disease (CAD). Ticagrelor blocks adenosine reuptake through the inhibition of equilibrative nucleoside transporter 1 (ENT-1) on erythrocytes and platelets, thereby facilitating adenosine-induced physiological responses such as an increase in coronary blood flow velocity. Meanwhile, adenosine plays an important role in triggering ischemic preconditioning through the activation of the A1 receptor. Therefore, an increase in ticagrelor-enhanced adenosine bioavailability may confer beneficial effects through mechanisms related to preconditioning activation and improvement of coronary microvascular dysfunction.

Methods: To determine whether ticagrelor can trigger ischemic preconditioning and influence microvascular function, we designed this prospective, open-label, pilot study that enrolled patients with stable multivessel CAD requiring staged, fractional flow reserve (FFR)-guided percutaneous coronary intervention (PCI). Participants will be randomized in 1:1 ratios either to ticagrelor (loading dose (LD) 180 mg, maintenance dose (MD) 90 mg bid) or to clopidogrel (LD 600 mg, MD 75 $\mathrm{mg}$ ) from 3 to 1 days before the scheduled $\mathrm{PCl}$. The $\mathrm{PCl}$ operators will be blinded to the randomization arm. The primary endpoint is the delta (difference) between ST segment elevations (in millimeters, $\mathrm{mm}$ ) as assessed by intracoronary electrocardiogram (ECG) during the two-step sequential coronary balloon inflation in the culprit vessel. Secondary endpoints are 1) changes in coronary flow reserve (CFR), index of microvascular resistance (IMR), and FFR measured in the culprit vessel and reference vessel at the end of $\mathrm{PCl}$, and 2) angina score during inflations. This study started in 2018 with the aim of enrolling 100 patients. Based on the rate of negative FFR up to $30 \%$ and a drop-out rate up to 10\%, we expect to detect an absolute difference of $4 \mathrm{~mm}$ among the study arms in the mean change of ST elevation following repeated balloon inflations. All study procedures were reviewed and approved by the Ethical Committee of the Catholic University of Sacred Heart.
\end{abstract}

Discussion: Ticagrelor might improve ischemia tolerance and microvascular function compared to clopidogrel, and these effects might translate to better long-term clinical outcomes.

Trial registration: EudraCT No. 2016-004746-28. No. NCT02701140.

(Continued on next page)

\footnotetext{
*Correspondence: italo.porto@gmail.com; filippo.crea@unicatt.it

${ }^{\dagger} \mathrm{D}$. D'Amario and I. Porto contributed equally to this work.

${ }^{4}$ Ospedale Policlinico San Martino IRCCS, Università degli Studi di Genova,

Genoa, Italy

${ }^{1}$ Fondazione Policlinico A. Gemelli IRCCS - Università Cattolica del Sacro

Cuore, Largo Agostino Gemelli 8, 00168 Rome, Italy

Full list of author information is available at the end of the article
}

(c) The Author(s). 2020 Open Access This article is distributed under the terms of the Creative Commons Attribution 4.0 International License (http://creativecommons.org/licenses/by/4.0/), which permits unrestricted use, distribution, and reproduction in any medium, provided you give appropriate credit to the original author(s) and the source, provide a link to the Creative Commons license, and indicate if changes were made. The Creative Commons Public Domain Dedication waiver (http://creativecommons.org/publicdomain/zero/1.0/) applies to the data made available in this article, unless otherwise stated. 


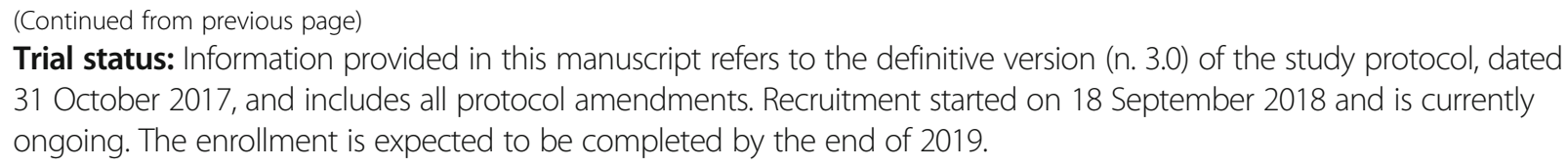

Keywords: Angina, Ischemic preconditioning, Ticagrelor, Clopidogrel, Adenosine, Fractional flow reserve, Intracoronary electrocardiography, Microvascular function, Microvascular dysfunction, Microvascular resistance

\section{Background \\ Rationale}

Ticagrelor is a reversibly binding, direct-acting, oral $\mathrm{P}_{2} \mathrm{Y}_{12}$ antagonist used for the prevention of atherothrombotic events in patients with acute coronary syndrome (ACS) [1]. It does not belong to thienopyridines; it is a carbocyclic nucleoside, representing a "first-in-class" cyclopentyl-triazolopyrimidine. In two phase II studies, dyspnea was noted to occur as a side effect to ticagrelor in a dose-dependent fashion, and in the PLATO study, a 6\% absolute excess of dyspnea was observed in ticagrelor-treated patients compared with patients treated with clopidogrel [1]. In the ONSET/ OFFSET study, dyspnea was more commonly associated with ticagrelor therapy in comparison with clopidogrel and placebo in patients with stable coronary artery disease (38.6\%, 9.3\%, and 8.3\%, respectively) but was not associated with any adverse change in cardiac or pulmonary function [2]. Similar results were later confirmed in the population of patients with ACS [3].

The mechanisms for this side effect are largely unknown, although early data indicate that ticagrelor blocks adenosine reuptake through inhibition of ENT-1 transporter by erythrocytes [4], and intravenous adenosine infusion can cause transient dyspnea in the absence of bronchoconstriction [5]. Another mechanism potentially increasing adenosine levels by ticagrelor is reflected in adenosine triphosphate (ATP) release from erythrocytes [6]. Moreover, the biochemical comparison between ticagrelor and adenosine suggests their molecular similarity. Adenosine is a well-known key endogenous molecule that regulates tissue functions by activating four G-protein-coupled adenosine receptors: A1, A2A, $\mathrm{A} 2 \mathrm{~B}$, and $\mathrm{A} 3$. Adenosine accumulates in the extracellular space in response to metabolic stress and cell damage, and elevations of adenosine are commonly encountered in conditions such as ischemia, hypoxia, inflammation, and trauma. Adenosine confers protective effects on the cell due to its anti-inflammatory, cardioprotective, cerebroprotective, antisclerotic, and antifibrotic properties, as well as by platelet inhibition and vasodilation [7].

Because to these effects of ticagrelor on adenosine biochemistry, chronic adenosine overload induced by ticagrelor was hypothesized to possibly contribute to the vascular outcome benefit observed in the PLATO trial, in addition to the inhibitory effect it has shown on platelet activity via $\mathrm{P}_{2} \mathrm{Y}_{12}$ receptor inhibition. Very recently, ticagrelor has been shown to increase adenosine-induced physiological responses in healthy human subjects by shifting the dose-response curve for adenosine-induced coronary blood flow velocity (CBFV) to the left and increasing area under the curve of CBFV [8]. Moreover, among patients with non-ST-segment elevation ACS that were treated with percutaneous coronary intervention (PCI) and received a maintenance dose of ticagrelor, a greater CBFV was observed compared to patients that received prasugrel maintenance dose in response to increasing adenosine concentrations [9]. These effects seem consistent with pharmacologically observed blockade of adenosine reuptake, as one of the pleiotropic effects exerted by the ticagrelor. An increase in adenosine bioavailability, as potentiated by ticagrelor, could have beneficial effects by both increasing coronary perfusion and enhancing the function of the microcirculation.

\section{Activation of preconditioning}

Ischemic preconditioning, consisting in episodes of ischemia as short as $5 \mathrm{~min}$, followed by reperfusion, has been showed to protect the heart from a subsequent longer coronary artery occlusion by markedly reducing the amount of necrosis. Adenosine plays a key role in triggering ischemic preconditioning. Indeed, the stimulation of A1 adenosine receptors triggers a complex pathway that includes the epsilon isoform of protein kinase C, the ATP-dependent potassium channels, and the mitochondrial permeability transition pores, as well as other cellular pathways, like a paradoxical protective release of oxygen radicals eventually making cardiomyocytes more resistant to ischemia $[10,11]$. In humans, examples of preconditioning are the preinfarction angina and the angina "warm-up" phenomenon. Preconditioning can be reproduced experimentally by repetitive and sequential balloon inflations in the culprit coronary artery during the $\mathrm{PCI}$, subsequently producing less chest pain and decrease in ST-segment elevation $[12,13]$.

Pharmacological preconditioning can be induced by intravenous or intracoronary administration of adenosine 
or A1 agonists of adenosine [14]. In a recent study performed in rabbits, investigators observed cardioprotective effects of clopidogrel and cangrelor (the intravenous analogue of ticagrelor) that were not attributed to the platelet inhibition but rather to activation of the signal transduction pathways of pre- and postconditioning, involving the reperfusion injury salvage kinases (RISK), including Akt and extracellular-regulated kinase (ERK), as well as adenosine A2B receptors, mitochondrial KATP channels, and redox signaling [15]. This cardioprotective effect of cangrelor was confirmed in a primate animal model as well $[16,17]$.

\section{Improvement of coronary microvascular dysfunction}

Coronary microvascular dysfunction has been demonstrated to affect the prognosis of ACS patients. Of note, Furber et al. described that doppler flow velocity parameters in the infarct-related artery are of prognostic value for long-term cardiac events [18]. Additionally, Takahashi et al. found an impaired coronary flow reserve velocity (CFVR) in the infarct-related artery to be significantly associated with increased cardiac event rates at long-term follow-up [19]. Furthermore, a microvascular function has been demonstrated to be altered even in nonischemic regions at distance from the infarcted myocardial tissue [20], and van de Hoef et al. have recently shown that microvascular dysfunction determined in the reference vessel after PCI is associated with a significantly increased long-term cardiac mortality [21].

Microvascular dysfunction is likely to occur also in the setting of non-ST-elevation ACS. In a cohort of 83 non-ST-elevation myocardial infarction (NSTEMI) patients, microvascular obstruction, as assessed by an elevated index of microcirculatory resistance (IMR) that is correlated with microvascular obstruction, was detected by the magnetic resonance imaging and was an independent predictor of adverse cardiovascular outcomes [22]. Marzilli et al. found that in patients with unstable angina, episodes of transient myocardial ischemia at rest are associated with a brisk increase in coronary microvascular resistance and that this increase is prevented by the administration of antiplatelet drugs [23]. Equally important, microvascular dysfunction may also occur following successful PCI; that is, coronary flow reserve has been shown to be impaired in the vascular bed subtended by the treated artery and requires up to 3 months for this microvascular dysfunction to resolve [24]. Finally, coronary microvascular reactivity to adenosine predicted adverse outcomes in women evaluated for suspected ischemia as demonstrated in WISE study [25].

\section{Pharmacology \\ Mechanism of action (taken from the SmPC of the drug in study)}

Ticagrelor, a member of the chemical class cyclopentyl triazolo pyrimidines (CPTP), is an oral, direct-acting, selective, and reversibly binding $\mathrm{P}_{2} \mathrm{Y}_{12}$ receptor antagonist that prevents adenosine diphosphate (ADP)-mediated $\mathrm{P}_{2} \mathrm{Y}_{12}$ dependent platelet activation and aggregation [26]. Ticagrelor does not prevent ADP binding but when bound to the $\mathrm{P}_{2} \mathrm{Y}_{12}$ receptor prevents ADP-induced signal transduction. Since platelets participate in the initiation and/or evolution of thrombotic complications of the atherosclerotic disease, inhibition of platelet function has been shown to reduce the risk of cardiovascular $(\mathrm{CV})$ events such as death, myocardial infarction, or stroke [27].

Ticagrelor also increases local endogenous adenosine levels by inhibiting the equilibrated nucleoside transporter1 (ENT-1). Ticagrelor has been documented to augment the following adenosine-induced effects in healthy subjects and in patients with ACS: vasodilation (measured by coronary blood flow increases in healthy volunteers and ACS patients), inhibition of platelet function (in human whole blood in vitro), and dyspnea [28, 29]. However, a link between the observed increases in adenosine and clinical outcomes (e.g., morbidity-mortality) has not been clearly elucidated $[11,30]$.

\section{Objectives}

The main goal of this study is to assess the pleiotropic effects of ticagrelor that could represent possible mechanisms for its beneficial effects on cardiovascular mortality and adverse outcomes.

\section{Primary objective}

Ticagrelor may trigger ischemic preconditioning as compared to clopidogrel in patients with stable multivessel CAD that are undergoing staged PCI.

\section{Secondary objectives}

Ticagrelor may improve microvascular perfusion in the myocardium of patients with stable multivessel CAD that are undergoing staged PCI.

\section{Study endpoints \\ Primary endpoint}

The primary endpoint is the comparison of ticagrelor and clopidogrel for the delta (difference in $\mathrm{mm}$ ) of the ST-segment elevation, as assessed by the intracoronary ECG during two-step sequential coronary balloon inflation in the index vessel.

\section{Secondary endpoints}

Secondary endpoints include the following: 
1) Comparison of ticagrelor and clopidogrel on CFR, IMR, and FFR measured in the index vessel at the end of the PCI

2) Comparison of ticagrelor and clopidogrel on angina score during coronary balloon inflation

\section{Materials/Methods}

TAPER-S trial design

This study is a prospective, randomized, blinded, endpoint trial that will enroll patients with multivessel CAD requiring scheduled treatment of a second lesion located in a nonculprit vessel. The typical patient will be a patient with purely stable CAD and a scheduled, FFRguided PCI of any vessel. Patient presenting initially with an ACS, with an intermediate coronary lesion (40-80\% in severity, where functional evaluation of the stenosis with FFR must be used based on clinical situation) in a nonculprit coronary artery, for whom a delayed, FFRguided strategy is chosen, may also be included in the study. In these patients, recovery of the microvascular function requires that at least 1 month has elapsed before re-evaluation of the culprit lesion, as FFR in the acute phase is known to be unreliable [31]. Screening/ randomization will take place 1 to 3 days before the index procedure. Randomization will be blocked within each study site, which will allow an even balance of patients to be randomized to either drug at each recruiting center. Patients with FFR $>0.80$ (no functionally significant stenosis) will be excluded from the study (Figs. 1 and 2). The study will be conducted in Italy, involving A. Gemelli Polyclinic of the Catholic University of the Sacred Heart in Rome and Grosseto Hospital (Ospedale della Misericordia Grosetto) in Grosetto, Italy.

The study is designed to have a total duration of 23 months, with an enrollment period of approximately 20 months. Each patient will be treated with the study drug only during the in-hospital phase, for a minimum of 3 days (Day -1 to Day 1) up to a maximum of 7 days (Day-1 to Day 5). The patient will be hospitalized (as per usual clinical practice) from Day -3 to Day -1 (admission) to Day $0-5$ of the study. The total number of enrolled patients will be one hundred $(N=100)$, with a projected $10 \%$ drop-out rate and $30 \%$ of negative FFR assessment $(F F R>0.80)$, with a final pool comprising 60 patients who will be evaluated in terms of primary and secondary endpoints. Therefore, the study enrollment will be stopped once one hundred patients are enrolled (Fig. 3).

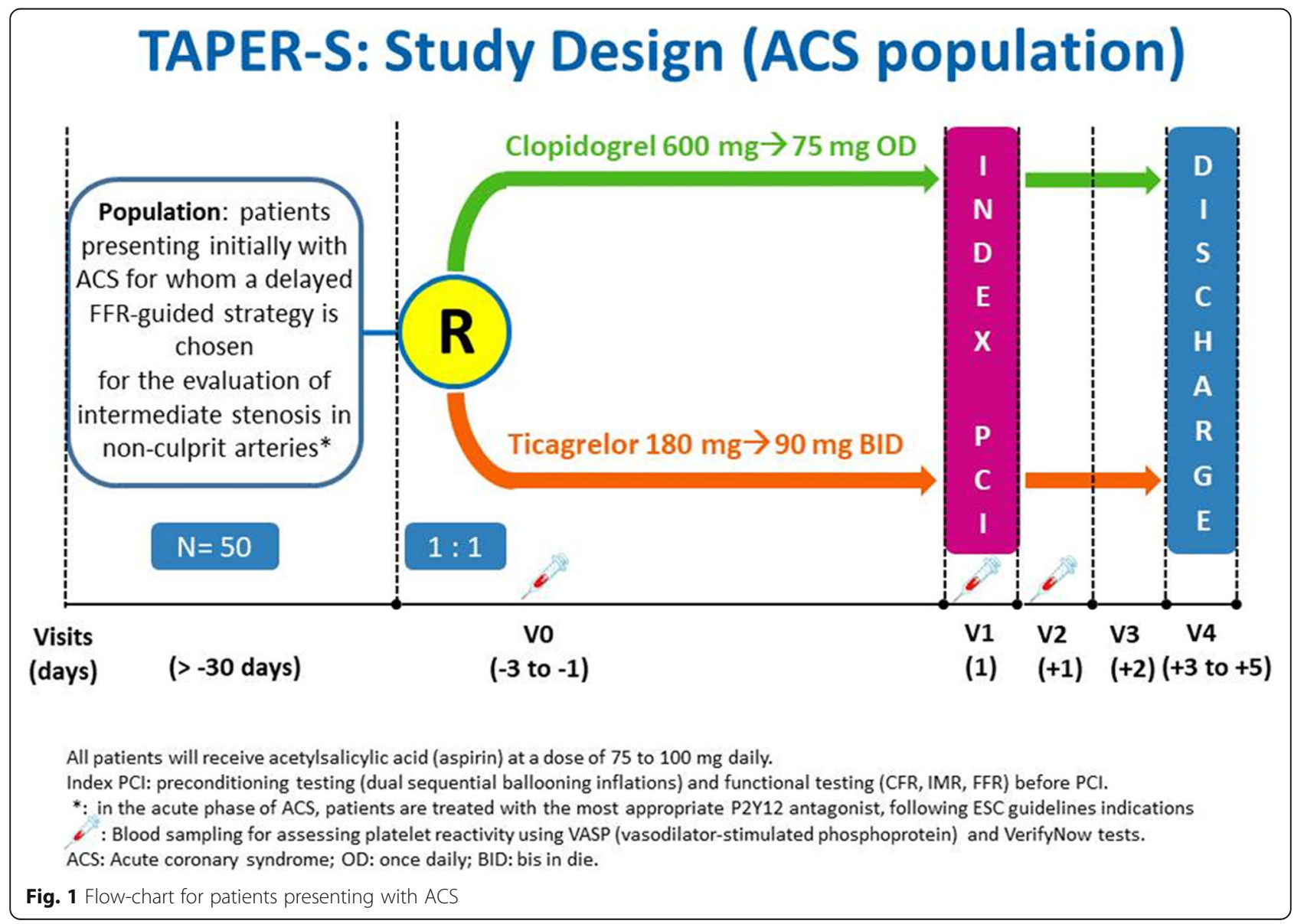




\section{TAPER-S: Study Design (stable population)}

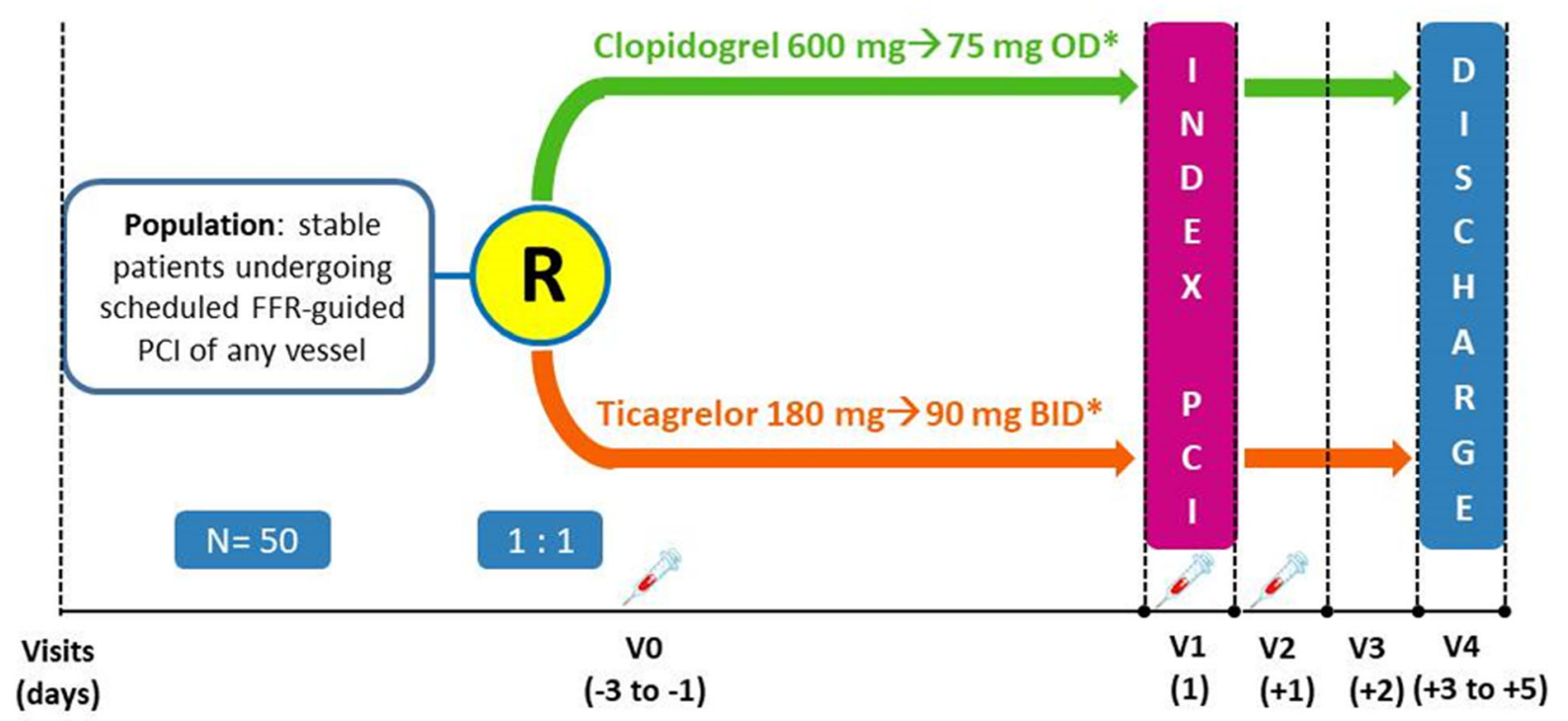

All patients will receive acetylsalicylic acid (aspirin) at a dose of 75 to $100 \mathrm{mg}$ daily. Loading dose will be used for naïve patients. Index $\mathrm{PCl}$ : preconditioning testing (dual sequential ballooning inflations) and functional testing (CFR, IMR, FFR) before PCI.

*: if the patient is already assuming the respective antiplatelet therapy, loading dose will be omitted.

: Blood sampling for assessing platelet reactivity using VASP (vasodilator-stimulated phosphoprotein) and VerifyNow tests. ACS: Acute coronary syndrome; OD: once daily; BID: bis in die.

Fig. 2 Flow-chart for patients presenting with stable coronary artery disease

\section{Sample size}

This is an exploratory and pilot study, aimed at evaluating the off-target effects of ticagrelor on ischemic preconditioning. A sample size of 40-60 patients is, in general, considered appropriate for a pilot study and should provide important insights for the planning of a future larger study and for estimating confidence intervals [32]. Per our design, by assuming an absolute difference (delta) of $4 \mathrm{~mm}$ in the change of ST-segment shift from the first to the second balloon inflation between the two compared groups, a magnitude similar (albeit reversed) to the effect observed by Niccoli et al. in a study demonstrating the negative preconditioning effect of ethanol [33], we can infer that 30 patients per group will be required to provide an $80 \%$ power to detect a statistically significant difference between groups at a significance level set at $p<0.05$. The standard deviation (SD) of the primary endpoint is expected to be approximately 5 to $4 \mathrm{~mm}$ in each treatment group. By enrolling one hundred patients, we also take into account those patients that will not require PCI after FFR evaluation (as observed in the FAME II trial) [34].

The main statistical analysis to be undertaken is a comparison of the primary endpoint (continuous variables) between the two groups of 30 patients each is a $2 \times 2$ analysis of variance (ANOVA) stratified by treatment group. The co-primary safety endpoint, which is a frequency value, will be analyzed using a Fisher's exact test.

\section{Study population}

Patients will be required to have a clinical indication to undergo scheduled FFR-guided PCI. Two types of patients will be included:

1) Patients presenting initially with an ACS, with an intermediate coronary lesion (40-80\% in severity, where functional evaluation with FFR must be used based on clinical situation) in a nonculprit artery, thus requiring a staged FFR-guided PCI (at least 1 month after the index ACS event)

2) Patients with a stable $C A D$ and who are undergoing scheduled FFR-guided PCI of any vessel

\section{Patient selection}

Inclusion criteria

The inclusion criteria are as follows: 


\section{Screening of 100 patients with CAD undergoing staged, FFR-guided PCI}

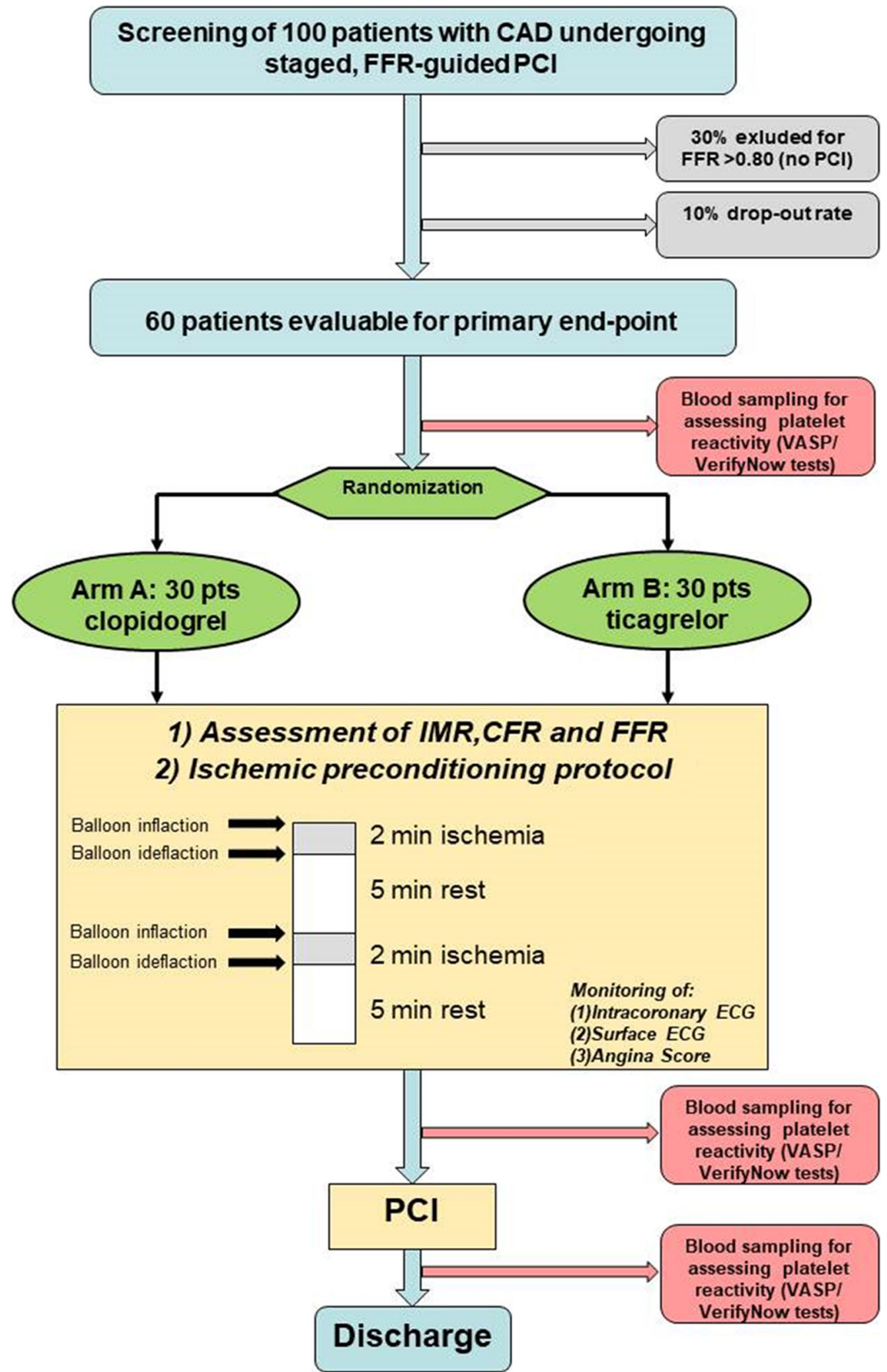

Fig. 3 Taper-S study flow-chart 
1) Signed and dated written Informed Consent obtained prior to the inclusion in the trial

2) Age $\geq 18$ and $\leq 75$ years

3) Weight $\geq 60 \mathrm{~kg}$

4) Patients must have a clinical indication to undergo scheduled FFR-guided PCI (the two types of patients to be included are noted in 5 and 6)

5) Patients presenting initially with an ACS, with an intermediate coronary lesion (40-80\% in severity, where functional evaluation with FFR must be used based on clinical situation) in a nonculprit artery, thus requiring a staged FFR-guided PCI (at least 1 month after the index ACS event);

6) Patients with a stable CAD undergoing scheduled FFR-guided PCI of any vessel

7) Patients already may be on the antiplatelet-directed treatment with either ticagrelor (previous ACS patients) or clopidogrel (stable patients)

\section{Exclusion criteria}

The exclusion criteria are as follows:

1) Known hypersensitivity to aspirin, clopidogrel, ticagrelor, or any similar drug

2) Concomitant oral anticoagulant therapy, or need for it (e.g., atrial fibrillation, mechanical valve, etc.)

3) Need for a concomitant cardiac noncoronary procedure, such as valve repair or replacement

4) Any previous history of ischemic stroke, intracranial hemorrhage or disease (neoplasm, arteriovenous malformation, or aneurysm)

5) Any active pathological bleeding or history of significant gastrointestinal bleeding, genitourinary bleeding or other site abnormal bleeding within the previous 3 months, other bleeding diathesis, or considered by the Investigator to be at high risk for bleeding

6) Concomitant oral or intravenous (IV) therapy with strong CYP3A inhibitors, CYP3A substrates with narrow therapeutic indices, or strong CYP3A inducers. Any of such concomitant treatment will be mapped and evaluated by the Investigator to comply with the exclusion criteria

7) Increased risk of bradycardia events

8) Known pregnancy and/or breast-feeding

9) Severe uncontrolled chronic obstructive pulmonary disease

10) Concomitant theophylline/aminophylline use

11) Baseline ECG with significant conduction abnormalities (i.e., left ventricular hypertrophy with repolarization abnormalities, left bundle branch block, etc.)
12) Evidence of prior myocardial infarction by cardiac imaging in the territory of the index vessel

13) Reduced left ventricular systolic function at screening/randomization (left ventricular ejection fraction $<40 \%$ within $48 \mathrm{~h}$ before index PCI, as assessed by transthoracic echocardiography)

14) Clinical and laboratory signs of congestive heart failure

15) Presence of coronary collaterals on diagnostic coronary angiography

16) Diffuse obstructive disease ( $\geq 70 \%$ stenosis) in the distal segment of the target vessel

17) Left main and/or three-vessel CAD

18) End-stage renal disease (as assessed by the estimated glomerular filtration rate CKD-EPI formula, eGFR $<15 \mathrm{~mL} / \mathrm{min} . / 1.73 \mathrm{~m}^{2}$ )

19) Documented severe hepatic impairment (e.g., elevated liver enzymes, cirrhosis, etc.)

\section{Study drugs}

\section{Dosing, posology, and route of administration}

Ticagrelor will be given in a loading dose of $180 \mathrm{mg}$ (on Day -3 to -1 ), followed by a dose of $90 \mathrm{mg}$ twice daily (from Day 0 to discharge between Days 1 to Day 5, during the index hospitalization). If the patient is chronically on ticagrelor, the loading dose will be omitted. In case of missed dose, the lapses in therapy should also be avoided. A patient who misses a dose of ticagrelor should take only one tablet (their next dose) at its scheduled time.

Clopidogrel will be given in a loading dose of 600 $\mathrm{mg}$ (on Days -3 to -1 ), followed by a dose of $75 \mathrm{mg}$ once a day (from Day 0 to discharge during Days 1 to Day 5, during the index hospitalization). If the patient is chronically on clopidogrel, the loading dose will be omitted. The antiplatelet switch will be managed according to generally agreed consensus. The details of the Antiplatelet Drug "switch" are specified in the section below.

\section{Antiplatelet drug switch}

Patients could be randomized while on treatment to either ticagrelor (previous ACS patients) or clopidogrel (stable patients). For those on long-term ticagrelor or clopidogrel treatment randomized to the corresponding drug, no loading dose will be given, and the patient will be continued on the respective drug. For those randomized to the noncorresponding group, the most recent recommendations regarding antiplatelet drug "switch" will be followed $[35,36]$ - a loading dose of the other drug will be given, 12-24 $\mathrm{h}$ before the scheduled PCI procedure. For patients previously on a long-term ticagrelor treatment, a 24-h washout period (i.e., the withdrawing of a single dose) will be applied before clopidogrel loading dose is initiated, to aid in clearance of the 
reversible platelet inhibition by ticagrelor and its main metabolite [36]. For both ticagrelor and clopidogrel, the last drug dose will be given at least $4 \pm 1 \mathrm{~h}$ before the start of the index procedure [37]. After the index procedure, longterm antiplatelet treatment will be administered according to the most recent guidelines [38]. Timing and schedule of all the procedures projected during the protocol visits are detailed in the flow chart table that is available below. See also Additional file 1 for details related to main pharmacological interactions and adverse events definition and reporting.

\section{Compliance}

Given that the administration of the drugs is conducted while the patients are hospitalized, the compliance to the treatment scheduled must be considered by default as $100 \%$ endorsed. Nevertheless, in case any of the requested doses are not to be administered, the issue and the relevant reason will be documented by the Investigator (and/ or his delegates) on the appropriate source document.

\section{Discontinuation/stopping of the treatment}

Enrolled subjects may be discontinued from the investigational product (IP) in case of consent withdrawal, any adverse event, and non-compliance to study protocol or incorrect enrollment.

\section{Data collection and management}

The referent and person responsible for the coordinating center, Fondazione Policlinico Universitario Agostino Gemelli, will be Italo Porto, MD, PhD. The source data, recorded in the appropriate source documentation, will be reported by the Principal Investigator and/or his delegates in a web-based database (electronic case report form, $\mathrm{eCRF}$ ). The validation of the inconsistencies (change or acceptance) will be made by the Principal Investigator and/ or his delegates. Before the data freezing, the person in charge of the data management will code the medical terms. Data will be collected by their registered delegates and will be stored in dedicated paper file, which will be locked for security. The electronic data (the eCRF) will be password secured.

\section{Inspections}

The Principal Investigator and/or his delegates must allow the regulatory authorities to conduct inspections. The inspection on the part of the Regulatory Authority consists of an official review of the documents, facilities, records, and any other resource considered by the authority to relate to the study. Auditing of the trial conduct will be performed according to the CTC Quality System and will be independent from investigators and the sponsor.

\section{Study procedures}

\section{Visit scheduling}

Patients satisfying inclusion criteria and having none of the exclusion criteria will be randomized to either ticagrelor or clopidogrel (Figs. 1 and 2). Ticagrelor will be administered in a loading dose of $180 \mathrm{mg}$ followed by a dose of $90 \mathrm{mg}$ twice daily. Clopidogrel will be administered in a loading dose of $600 \mathrm{mg}$, followed by a dose of $75 \mathrm{mg}$ once a day. If the patient is chronically on ticagrelor or clopidogrel treatment, the loading dose will be omitted.

All patients will receive acetylsalicylic acid (aspirin) at a dose of 75 to $100 \mathrm{mg}$ daily. For those who had not previously been receiving aspirin, 250 or $325 \mathrm{mg}$ will be the preferred loading dose. The study drug will be continued for at least $72 \mathrm{~h}$ before the second PCI.

During PCI, two sequential balloon inflations will be performed to measure ischemic preconditioning. Operators will measure the degree of ST-segment elevation by intracoronary ECG and by surface ECG during coronary balloon inflation.

\section{Randomization}

Patients will be randomly assigned to ARM A (clopidogrel) or ARM B (ticagrelor) in a 1:1 ratio. The allocation to the assigned treatment arm will be known to the patients and to the Principal Investigator (PI) and/or his delegates.

The randomization will be performed at Visit 0 . Randomization process will be performed printing 50 tags reporting the word "Ticagrelor" and 50 tags reporting the word "Clopidogrel" inserted into anonymous white letter envelopes; the envelopes will be mixed and then stacked up in random order, with the opening side toward the bottom of the pile, by the study monitor; the resulting random sequence of 100 envelopes will be consigned to the PI or his delegates, which are entrusted with their preservation and responsible for any adulteration. At visit 0 the PI or his delegates will open the first available envelope, proceeding from top to bottom of the pile. The already opened envelopes will be stored separately from the others and will be available to the study monitor on request.

\section{Study timeline}

The study timeline is depicted in Table 1 . See also the checklist enclosed in Additional file 2.

\section{Assessments description Dyspnea evaluation}

The sensation of dyspnea will be reported by patients using the modified Borg scale; the scale is scored from 1 
(no sensation of dyspnea) to 10 (maximum sensation of dyspnea) [39].

\section{Assessment of platelet reactivity}

We will perform platelet VASP (vasodilator-stimulated phosphoprotein) and VerifyNow test. The VASP test specifically assesses the inhibition of platelet reactivity index (PRI) linked to $\mathrm{P}_{2} \mathrm{Y}_{12}$ ADP receptor inhibition. The VASP test is a flow cytometry-based method that uses a phosphorylation-specific antibody and measures the PRI, expressed as a percentage and defined as the difference in VASP fluorescence intensity between resting (+PGE1, prostaglandin E1) and activated (+ADP) platelets. VASP phosphorylation analysis can evaluate the individual response to the clopidogrel loading dose prior to PCI and predict post-procedural major adverse cardiac events (MACE). In a study of Bonello et al., the optimal cut-off value of PRI assessed by VASP to exclude adverse events was $50 \%$ $[40,41]$.

VerifyNow $\mathrm{P}_{2} \mathrm{Y}_{12}$ test (Accumetrics, Inc., San Diego, CA) measures the agglutination of fibrinogen-coated beads by platelets stimulated by an agonist (ADP with prostaglandin E1) in citrated whole blood. The relevant thresholds are not clearly defined. Each assay records two results: $\mathrm{P}_{2} \mathrm{Y}_{12}$ reaction units (PRU) and percentage of inhibition. A good correlation has been established between the VerifyNow $\mathrm{P}_{2} \mathrm{Y}_{12}$ test and several other methods including the VASP assay [42].

Two blood samples for PRI and PRU testing will be drawn in Vacutainer tubes containing 3.8\% trisodium citrate. The first sample will be collected at baseline following the randomization and then just before the index procedure. The analysis and the interpretation will be carried out in the Department of Cardiovascular Medicine of A. Gemelli Polyclinic or in the Grosseto Hospital according to the site of enrollment. The authors will store any residual blood sample for future research purposes.

For evaluation of the adenosine-induced effect of ticagrelor compared to clopidogrel, left-overs from the blood sampling will be utilized to measure the adenosine plasma concentration in the two groups together with ENT-1 expression and activity.

\section{Ischemic preconditioning}

During PCI, two sequential balloon inflations will be performed to measure preconditioning. Operators will measure the degree of ST-segment elevation by intracoronary ECG and by surface ECG during coronary balloon inflation.

Specifically, after passage of the guidewire through a target stenosis and then advancement of the balloon catheter across the stenosis, the intracoronary unipolar ECG from myocardium distal to the stenosis to be dilated will be obtained, connecting the proximal end of the guidewire as it exited from the balloon catheter. The unipolar intracoronary ECG will be filtered between 0.1 to 100 or 0.1 to 500 $\mathrm{Hz}$ and then displayed simultaneously with the standard surface leads being monitored. Surface and intracoronary ECGs will be recorded simultaneously and continuously during balloon inflation and deflation [43-45].

Moreover, angina score during coronary balloon inflation will be registered by using a Visual Analog Scale (VAS) on a scale of 0 (no pain) to 10 (most severe pain) [46]. For pharmacodynamic considerations, the Index PCI will be performed at $4 \pm 1 \mathrm{~h}$ after ticagrelor or clopidogrel last dose.

\section{Quantification of coronary microvascular resistance}

CFR and IMR will be measured with an intracoronary pressure/temperature sensor-tipped guidewire (Radi pressure wire 4, Radi Medical Systems, Wilmington, Mass., USA), with an accuracy of $0.05^{\circ} \mathrm{C}$ within a temperature range of $15-42^{\circ} \mathrm{C}$, to derive thermodilution curves. Previous experimental and human studies have demonstrated that the mean transit time (Tmn) of an intracoronary injection of saline at room temperature, derived from thermodilution curves, is inversely proportional to coronary flow [47-49]. Therefore, a given percent decrease in Tmn closely reflects a proportional percent increase in coronary blood flow. Three injections ( $3 \mathrm{~mL}$ each) of saline at room temperature will be performed, and the resting Tmn will be measured. An intravenous infusion of adenosine (Adenoscan, Sanofi Aventis S.p.A, Milan, Italy) at $140 \mu \mathrm{g} / \mathrm{kg} / \mathrm{min}$ will be administered to induce a steady-state maximal hyperemia, followed by three further injections $(3 \mathrm{~mL})$ of room temperature saline to measure hyperemic Tmn.

Simultaneous measurements of mean aortic pressure ( $\mathrm{Pa}$, through the guiding catheter) and mean distal coronary pressure (Pd, by the pressure wire) will also be obtained in the resting and maximal hyperemic states. The following parameters will be calculated:

1. CFR will be calculated as the resting Tmn divided by the hyperemic Tmn $[47,48]$

2. IMR will be calculated as the distal coronary pressure at maximal hyperemia divided by the inverse of the hyperemic Tmn [50]

3. BR (Baseline resistance index) will be calculated using the equation: $\mathrm{BR}=$ Pabase $\times$ Tmnbase $($ Pdbase $-\mathrm{Pw}) /($ Pabase $-\mathrm{Pw})[51]$

4. RRR (resistive reserve ratio) will be calculated using the following equation: $R R R=B R$ index / IMR $[49,50]$ 
Table 1 A TAPER-S study timeline

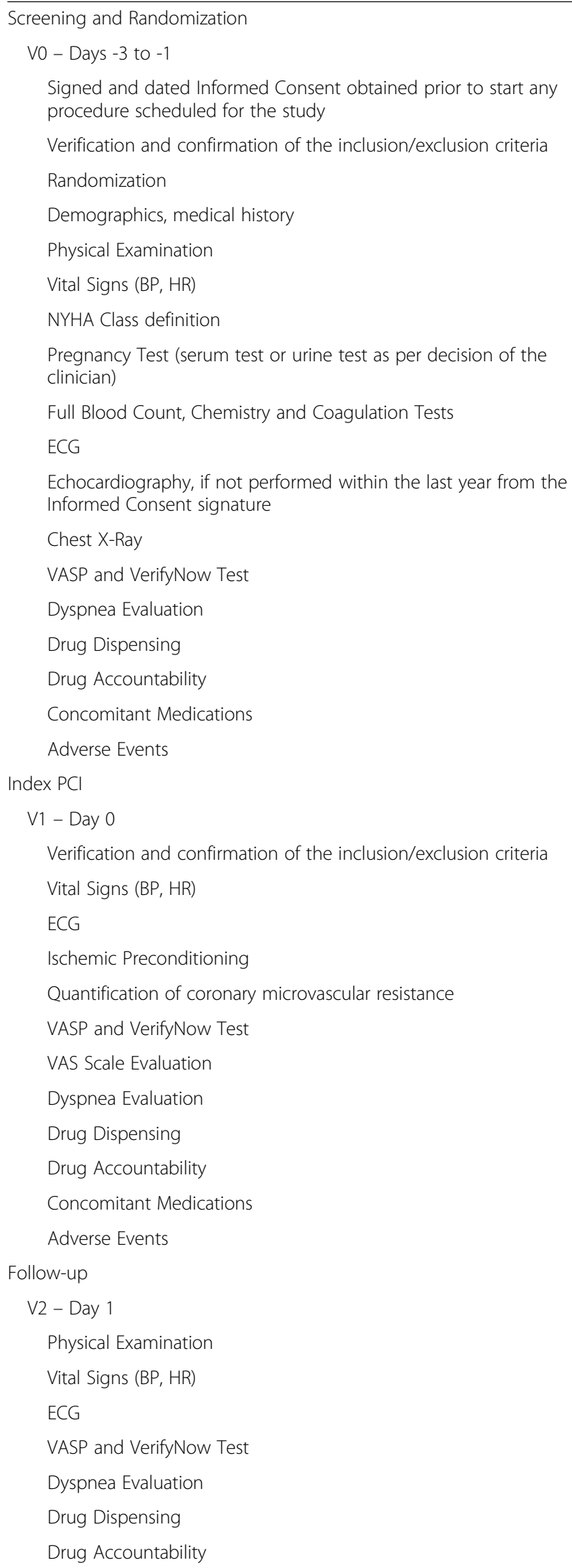

Table 1 A TAPER-S study timeline (Continued)

\begin{tabular}{l} 
Concomitant Medications \\
Adverse Events \\
Pre-discharge Visit \\
V3 - Day 2 \\
Physical Examination \\
Vital Signs (BP, HR) \\
Dyspnea Evaluation \\
Drug Dispensing \\
Drug Accountability \\
Concomitant Medications \\
Adverse Events \\
Discharge/End of Study Visit \\
V4 - Day 3 to Day 5 \\
Physical Examination \\
Vital Signs (BP, HR) \\
ECG \\
Full Blood Count, Chemistry and Coagulation Tests \\
Dyspnea Evaluation \\
Drug Dispensing \\
Drug Accountability \\
Concomitant Medications \\
Adverse Events \\
\hline
\end{tabular}

\section{Statistical analysis}

All the variables will be descriptively analyzed by arm and visit (mean, median, standard deviation, minimum and maximum for continuous variables, and frequency distribution for categorical variables). Categorical variables referring to a physical examination, vital signs and laboratory abnormalities will also be analyzed with shift tables (baseline vs. final visit). All analyses will be carried out in details in the respective Statistical Analysis Plan (SAP), which will be finalized in Version 1.0 before the database freezing.

Version 2.0 of the SAP will be prepared after the Data Review Meeting (in which the protocol violations will be evaluated) and will include the list of the patients belonging to the defined populations and the randomization list.

A descriptive analysis of demographic data and baseline characteristics will be carried out to verify the balance of the two arms at the study entry.

The delta ST-segment elevation by intracoronary ECG during two-step sequential coronary balloon inflation in the culprit vessel will be compared between the ticagrelor and clopidogrel arms by using two-way ANOVA $(2 \times 2)$. Missing data in the primary analysis will be handled by simple imputations. An interim efficacy analyses 
will be conducted after the accrual of two-third of therandomized patients have been reached.

\section{Supplementary information}

Supplementary information accompanies this paper at https://doi.org/10. 1186/s13063-020-4116-7.

Additional file 1. Details related to main pharmacological interactions and adverse events definition and reporting are provided in the additional file, enclosed in the manuscript.

Additional file 2. SPIRIT 2013 Checklist.

\section{Abbreviations}

ACS: Acute coronary syndrome; AE: Adverse events; AIFA: Agenzia Italiana del Farmaco; ANOVA: Analysis of variance; BP: Blood pressure; BR: Baseline resistance index; CAD: Coronary artery disease; CBFV: Coronary blood flow velocity; CFR: Coronary flow reserve; CFVR: Coronary flow reserve velocity; CTC: Clinical Trial Center S.p.A.; DAPT: Dual antiplatelet therapy; EC: Ethics committee; eCRF: Electronic case report form; EMA: European Medicines Agency; FFR: Fractional flow reserve; FPG: Fondazione Policlinico Universitario A. Gemelli; HR: Heart rate; IC: Informed consent; ICH: International Conference on Harmonization; IMR: Index of microvascular resistance; MACE: Major adverse cardiac events; NSTEMI: Non-ST-elevation Acute Coronary Syndromes; Pa: Aortic pressure; PCl: Percutaneous coronary intervention; Pd: Distal coronary pressure; PRI: Platelet reactivity index PRU: P2Y 12 reaction units; PW: Wedge pressure; RISK: Reperfusion injury salvage kinases; RRR: Resistive reserve ratio; SAE: Serious adverse event; SD: Standard deviation; SUSAR: Suspected unexpected serious adverse reactions: $T_{m n}$ : Transit time; VASP: Vasodilator-stimulated phosphoprotein

\section{Acknowledgements}

None.

\section{Authors' contributions}

DDA and IP conceived and designed the study. The protocol was amended and critically revised by $A R, F C, A M L, S M, R V, M G, C T, F B, C A, G N, A B, R A M$, $A F, F F, F F r, G T, U L, J A B$, and FC. All authors read and approved the final manuscript.

\section{Funding}

The Principal sponsor of this study is Fondazione Policlinico Universitario A Gemelli - Roma, Polo di Scienze Cardiovascolari e Toraciche. The study will be partially supported by a grant provided by AstraZeneca, in a financial magnitude that is in accordance with the requirements of the acceptable funding as regulated by the Italian law on Non-Profit Studies ("Decreto 17 Dicembre 2004"). This sponsor will not have any role in the collection, management, analysis, and interpretation of the data.

\section{Availability of data and materials}

The datasets used and/or analyzed during the current study are available from the corresponding author on reasonable request. Case report forms and study documents will be electronically implemented and filled in at any visit. Data will be protected by passwords assigned to the PI and his registered delegates. The Institution Fondazione Policlinico Universitario A. Gemelli, being the Sponsor of the presented study, and represented by Dr. Italo Porto as a Principal Investigator (as per contract stipulated with AstraZeneca and IP) has the full ownership of the results and all accompanying data. Study investigators and collaborators are committed to publish obtained results publicly in a peer-reviewed biomedical journal. The final trial dataset will only be available to principal site investigators and will not be shared/disclosed to any third party or auxiliary trial sponsors (such as AstraZeneca that provided partial grant for this study). The full study protocol will be published and made available on ClinicalTrials.gov (https://clinicaltrials.gov). All personal data will be collected, stored, shared, and maintained according to EU Regulations and local laws.

\section{Ethics approval and consent to participate}

The study has been independently reviewed and approved by the respective Ethics Committee of A. Gemelli Polyclinic of the Catholic University of the
Sacred Heart in Rome, Italy (definitive approval 23 Nov. 2017, prot. No. 48133/17, ID: 1605; AIFA approval 21 Aug. 2017, prot. No. AIFA/SC/P/90531). All patients will be voluntarily included in this trial and will be officially enrolled once the informed written consent has been signed. The Principal Investigator or his delegates are responsible for obtaining a written informed consent from each subject. The Principal Investigator or his delegates will explain the nature of the trial, its purpose, the procedures involved, the expected duration, the potential risks and benefits involved, and any discomfort it may entail and provide the subject with a copy of the Informed Consent fully signed. The subject will be given sufficient time to consider the trial before deciding whether to participate. Each subject must be informed that participation in the trial is voluntary and that he/she may withdraw from the trial at any time and that withdrawal of consent will not affect his/her subsequent medical treatment or relationship with the treating physician. All patients are to provide written informed consent in accordance with applicable laws of the country. The patient will sign and date the informed consent form before he/she enters the study (i.e., before any study-related activity). The Principal Investigator or his delegates will explain the nature of the study, its purpose and the expected duration. The patient will be given sufficient time to consider the study before deciding whether to participate. Each patient must be informed that participation in the study is voluntary and that he/she may withdraw from the study at any time and that withdrawal of consent will not affect his/her medical treatment or relationship with the treating Investigator. In the case of an amendment that would directly affect the patient's participation in the study, the patient must provide new written informed consent indicating that he/she re-consents to participate in the study.

This trial will be carried out in compliance with the protocol, designed to ensure adherence to Good Clinical Practice, as described:

1. ICH Harmonised Tripartite Guidelines for Good Clinical Practice, 1996. Note for Guidance on Good Clinical Practice CPMP/ICH/135/95 (and subsequent revisions).

2. EU Directive 2001/20/EC, 2005/28/EC and its receipt in local laws.

3. Declaration of Helsinki (1964, and its amendments and subsequent clarification)

The Principal Investigator agrees, when signing the protocol, to adhere to the instructions and procedures described in it and thereby to adhere to the principles of Good Clinical Practice to which it conforms. To the same extent, any of his delegates will be bound to the same compliance.

Any protocol modifications such as changes to eligibility criteria, outcomes, pre-specified analyses, etc. will be disclosed to relevant parties, respective Ethics Committees at both participating centers, trial participants, and the trial registry in which this study has been registered.

Personal information of potential and enrolled participants will be collected at the dedicated computer system and will be password-protected and available only to site investigators. Confidentially of patients will be protected before, during, and after the trial. Fondazione Policlinico Universitario Agostino Gemelli will have access to the final trial dataset.

\section{Consent for publication}

Not applicable.

\section{Competing interests}

The authors declare that they have no competing interests.

\section{Author details}

${ }^{1}$ Fondazione Policlinico A. Gemelli IRCCS - Università Cattolica del Sacro Cuore, Largo Agostino Gemelli 8, 00168 Rome, Italy. Dipartimento Cardio neuro vascolare, Azienda USL Toscana Sud-est, Ospedale di Grosseto, Grosseto, Italy. ${ }^{3}$ Department of Pathophysiology, University of Split School of Medicine (USSM) and University Hospital Center Split (UHC Split), Split, Croatia. ${ }^{4}$ Ospedale Policlinico San Martino IRCCS, Università degli Studi di Genova, Genoa, Italy.

Received: 4 April 2019 Accepted: 29 January 2020

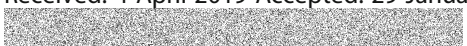

\section{References}

1. Wallentin L, Becker RC, Budaj A, Cannon CP, Emanuelsson H, Held C, et al. Ticagrelor versus clopidogrel in patients with acute coronary syndromes. N Engl J Med. 2009;361(11):1045-57. 
2. Storey RF, Bliden KP, Patil SB, Karunakaran A, Ecob R, Butler K, et al. Incidence of dyspnea and assessment of cardiac and pulmonary function in patients with stable coronary artery disease receiving ticagrelor, clopidogrel, or placebo in the ONSET/OFFSET study. J Am Coll Cardiol. 2010;56(3):185-93.

3. Storey RF, Becker RC, Harrington RA, Husted S, James SK, Cools F, et al. Pulmonary function in patients with acute coronary syndrome treated with ticagrelor or clopidogrel (from the Platelet Inhibition and Patient Outcomes [PLATO] pulmonary function substudy). Am J Cardiol. 2011;108(11):1542-6.

4. van Giezen JJ, Sidaway J, Glaves P, Kirk I, Bjorkman JA. Ticagrelor inhibits adenosine uptake in vitro and enhances adenosine-mediated hyperemia responses in a canine model. J Cardiovasc Pharmacol Ther. 2012;17(2):164-72.

5. Burki NK, Dale WJ, Lee LY. Intravenous adenosine and dyspnea in humans. J Appl Physiol (1985). 2005;98(1):180-5.

6. Ohman J, Kudira R, Albinsson S, Olde B, Erlinge D. Ticagrelor induces adenosine triphosphate release from human red blood cells. Biochem Biophys Res Commun. 2012;418(4):754-8.

7. Hale SL, Kloner RA. Cardioprotection with adenosine-regulating agent, GP531: effects on no-reflow, infarct size, and blood flow following ischemia/ reperfusion in the rabbit. J Cardiovasc Pharmacol Ther. 2010;15(1):60-7.

8. Wittfeldt A, Emanuelsson H, Brandrup-Wognsen G, van Giezen JJ, Jonasson J, Nylander S, et al. Ticagrelor enhances adenosine-induced coronary vasodilatory responses in humans. J Am Coll Cardiol. 2013;61(7):723-7.

9. Alexopoulos D, Moulias A, Koutsogiannis N, Xanthopoulou I, Kakkavas A, Mavronasiou $E$, et al. Differential effect of ticagrelor versus prasugrel on coronary blood flow velocity in patients with non-ST-elevation acute coronary syndrome undergoing percutaneous coronary intervention: an exploratory study. Circ Cardiovasc Interv. 2013;6(3):277-83.

10. Tomai F, Crea F, Chiariello L, Gioffre PA. Ischemic preconditioning in humans: models, mediators, and clinical relevance. Circulation. 1999;100(5): 559-63

11. Layland J, Carrick D, Lee M, Oldroyd K, Berry C. Adenosine: physiology, pharmacology, and clinical applications. J Am Coll Cardiol. 2014;7(6):581-91.

12. Kloner RA, Rezkalla SH. Preconditioning, postconditioning and their application to clinical cardiology. Cardiovasc Res. 2006;70(2):297-307.

13. Floyd JS, Maynard C, Weston P, Johanson P, Jennings RB, Wagner GS. Effects of ischemic preconditioning and arterial collateral flow on ST-segment elevation and QRS complex prolongation in a canine model of acute coronary occlusion. J Electrocardiol. 2009;42(1):19-26.

14. Tomai F, Crea F, Gioffre PA. Preconditioning, collateral recruitment and adenosine. J Am Coll Cardiol. 2000;35(1):259-60.

15. Yang XM, Liu Y, Cui L, Yang X, Liu Y, Tandon N, et al. Platelet P2Y(1)(2) blockers confer direct postconditioning-like protection in reperfused rabbit hearts. J Cardiovasc Pharmacol Ther. 2013;18(3):251-62.

16. Tissier R, Cohen MV, Downey JM. Protecting the acutely ischemic myocardium beyond reperfusion therapies: Are we any closer to realizing the dream of infarct size elimination? Arch Mal Coeur Vaiss. 2007;100(9): 794-802.

17. Yang XM, Liu Y, Cui L, Yang X, Liu Y, Tandon N, et al. Two classes of antiplatelet drugs reduce anatomical infarct size in monkey hearts. Cardiovasc Drugs Ther. 2013;27(2):109-15.

18. Furber AP, Prunier F, Nguyen HC, Boulet S, Delepine S, Geslin P. Coronary blood flow assessment after successful angioplasty for acute myocardial infarction predicts the risk of long-term cardiac events. Circulation. 2004;110(23):3527-33.

19. Takahashi T, Hiasa Y, Ohara Y, Miyazaki S, Ogura R, Miyajima H, et al. Usefulness of coronary flow reserve immediately after primary coronary angioplasty for acute myocardial infarction in predicting long-term adverse cardiac events. Am J Cardiol. 2007;100(5):806-11.

20. Bax M, de Winter RJ, Koch KT, Schotborgh CE, Tijssen JG, Piek JJ. Time course of microvascular resistance of the infarct and noninfarct coronary artery following an anterior wall acute myocardial infarction. Am J Cardiol. 2006;97(8):1131-6.

21. van de Hoef TP, Bax M, Meuwissen M, Damman P, Delewi R, de Winter RJ, et al. Impact of coronary microvascular function on long-term cardiac mortality in patients with acute ST-segment-elevation myocardial infarction. Circ Cardiovasc Interv. 2013;6(3):207-15.

22. Held EP, Henry TD. When small vessels become big problems! Microvascular dysfunction in NSTEMI. Catheter Cardiovasc Interv. 2018;92(6):1075-6.

23. Marzilli M, Sambuceti G, Testa R, Fedele S. Platelet glycoprotein Ilb/lla receptor blockade and coronary resistance in unstable angina. J Am Coll Cardiol. 2002;40(12):2102-9.
24. Uren NG, Crake T, Lefroy DC, de Silva R, Davies GJ, Maseri A. Delayed recovery of coronary resistive vessel function after coronary angioplasty. J Am Coll Cardiol. 1993;21(3):612-21.

25. Pepine CJ, Anderson RD, Sharaf BL, Reis SE, Smith KM, Handberg EM, et al. Coronary microvascular reactivity to adenosine predicts adverse outcome in women evaluated for suspected ischemia results from the National Heart, Lung and Blood Institute WISE (Women's Ischemia Syndrome Evaluation) study. J Am Coll Cardiol. 2010;55(25):2825-32.

26. Rognoni A, Cavallino C, Lupi A, Secco GG, Veia A, Bacchini S, et al. Ticagrelor: a novel drug for an old problem. Recent Pat Cardiovasc Drug Discov. 2014;9(1):51-7.

27. Papapanagiotou A, Daskalakis G, Siasos G, Gargalionis A, Papavassiliou AG. role of platelets in cardiovascular disease: molecular mechanisms. Curr Pharm Des. 2016;22(29):4493-505.

28. Kubisa MJ, Jezewski MP, Gasecka A, Siller-Matula JM, Postuła M. Ticagrelor toward more efficient platelet inhibition and beyond. Ther Clin Risk Manag. 2018;14:129-40.

29. Danielak D, Karazniewicz-Lada M, Glowka F. Ticagrelor in modern cardiology - an up-to-date review of most important aspects of ticagrelor pharmacotherapy. Expert Opin Pharmacother. 2018;19(2):103-12.

30. Mustafa SJ, Morrison RR, Teng B, Pelleg A. Adenosine receptors and the heart: role in regulation of coronary blood flow and cardiac electrophysiology. Handb Exp Pharmacol. 2009;193:161-88.

31. Hakeem A, Edupuganti MM, Almomani A, Pothineni NV, Payne J, Abualsuod AM, et al. Long-term prognosis of deferred acute coronary syndrome lesions based on nonischemic fractional flow reserve. J Am Coll Cardiol. 2016;68(11):1181-91.

32. Thabane L, Ma J, Chu R, Cheng J, Ismaila A, Rios LP, et al. A tutorial on pilot studies: the what, why and how. BMC Med Res Methodol. 2010;10:1.

33. Niccoli G, Altamura L, Fabretti A, Lanza GA, Biasucci LM, Rebuzzi AG, et al. Ethanol abolishes ischemic preconditioning in humans. J Am Coll Cardiol. 2008;51(3):271-5.

34. De Bruyne B, Pijls NHJ, Kalesan B, Barbato E, Tonino PAL, Piroth Z, et al. Fractional flow reserve-guided pci versus medical therapy in stable coronary disease. N Engl J Med. 2012;367(11):991-1001.

35. De Luca L, Capranzano P, Patti G, Parodi G. Switching of platelet P2Y12 receptor inhibitors in patients with acute coronary syndromes undergoing percutaneous coronary intervention: Review of the literature and practical considerations. Am Heart J. 2016;176:44-52.

36. Rollini F, Franchi F, Angiolillo DJ. Switching P2Y12-receptor inhibitors in patients with coronary artery disease. Nat Rev Cardiol. 2016;13(1):11-27.

37. Rollini F, Franchi F, Singh $K$, Cho JR, Bhatti M, DeGroat C, et al. Impact of timing from blood sampling to pharmacodynamic assessment on measures of platelet reactivity in patients treated with $\mathrm{P} 2 \mathrm{Y} 12$ receptor inhibitors. Thromb Haemost. 2016;116(6):1060-9.

38. Park Y, Franchi F, Rollini F, Angiolillo DJ. Dual antiplatelet therapy after coronary stenting. Expert Opin Pharmacother. 2016;17(13):1775-87.

39. Stenton C. The MRC breathlessness scale. Occup Med. 2008;58(3):226-7.

40. Aleil B, Ravanat C, Cazenave JP, Rochoux G, Heitz A, Gachet C. Flow cytometric analysis of intraplatelet VASP phosphorylation for the detection of clopidogrel resistance in patients with ischemic cardiovascular diseases. J Thromb Haemost. 2005;3(1):85-92.

41. Bonello L, Paganelli F, Arpin-Bornet M, Auquier P, Sampol J, Dignat-George $F$, et al. Vasodilator-stimulated phosphoprotein phosphorylation analysis prior to percutaneous coronary intervention for exclusion of postprocedural major adverse cardiovascular events. J Thromb Haemost. 2007;5(8):1630-6.

42. Campo G, Valgimigli M, Frangione A, Luccarelli S, Cangiano E, Cavazza C, et al. Evaluation of platelet inhibition by tirofiban in patients stratified according to aspirin and clopidogrel responsiveness: the 3T/2R (Tailoring treatment with tirofiban in patients showing resistance to aspirin and/or resistance to clopidogrel). J Am Coll Cardiol. 2010;55(3):255-6.

43. Friedman PL, Shook TL, Kirshenbaum JM, Selwyn AP, Ganz P. Value of the intracoronary electrocardiogram to monitor myocardial ischemia during percutaneous transluminal coronary angioplasty. Circulation. 1986;74(2):330-9.

44. Tomai F, Crea F, Danesi A, Perino M, Gaspardone A, Ghini AS, Ruggeri G, Chiariello L, Gioffrè PA. Effects of A1 adenosine receptor blockade on the warm-up phenomenon. Cardiologia. 1997;42(4):385-92.

45. Tomai F, Crea F, Gaspardone A, Versaci F, De Paulis R, Polisca P, Chiariello L, Gioffrè PA. Effects of A1 adenosine receptor blockade by bamiphylline on ischaemic preconditioning during coronary angioplasty. Eur Heart J. 1996; 17(6):846-53. 
46. Huskisson EC. Measurement of pain. Lancet. 1974;2(7889):1127-31.

47. De Bruyne B, Pijls NH, Smith L, Wievegg M, Heyndrickx GR. Coronary thermodilution to assess flow reserve: experimental validation. Circulation. 2001:104(17):2003-6.

48. Pijls NH, De Bruyne B, Smith L, Aarnoudse W, Barbato E, Bartunek J, et al. Coronary thermodilution to assess flow reserve: validation in humans. Circulation. 2002;105(21):2482-6.

49. Fearon WF, Farouque HM, Balsam LB, Caffarelli AD, Cooke DT, Robbins RC, et al. Comparison of coronary thermodilution and Doppler velocity for assessing coronary flow reserve. Circulation. 2003;108(18):2198-200.

50. Fearon WF, Balsam LB, Farouque HM, Caffarelli AD, Robbins RC, Fitzgerald $\mathrm{PJ}$, et al. Novel index for invasively assessing the coronary microcirculation. Circulation. 2003;107(25):3129-32.

51. Layland J, Carrick D, McEntegart M, Ahmed N, Payne A, McClure J, et al. Vasodilatory capacity of the coronary microcirculation is preserved in selected patients with non-ST-segment-elevation myocardial infarction. Circ Cardiovasc Interv. 2013;6(3):231-6.

\section{Publisher's Note}

Springer Nature remains neutral with regard to jurisdictional claims in published maps and institutional affiliations.

Ready to submit your research? Choose BMC and benefit from:

- fast, convenient online submission

- thorough peer review by experienced researchers in your field

- rapid publication on acceptance

- support for research data, including large and complex data types

- gold Open Access which fosters wider collaboration and increased citations

- maximum visibility for your research: over $100 \mathrm{M}$ website views per year

At $\mathrm{BMC}$, research is always in progress.

Learn more biomedcentral.com/submissions 